\title{
INTRODUCTION. LA SÉGRÉGATION MISE EN MOTS : QUELQUES RÉFLEXIONS SUR LA DIMENSION SOCIOLINGUISTIQUE DU PROCESSUS
}

\author{
Gudrun Ledegen et Thierry Bulot
}

L'Harmattan | «Cahiers internationaux de sociolinguistique »

2013/1 Nº 3 | pages 7 à 12

ISSN 2257-6517

ISBN 9782343010311

Article disponible en ligne à l'adresse :

https://www.cairn.info/revue-cahiers-internationaux-desociolinguistique-2013-1-page-7.htm

Distribution électronique Cairn.info pour L'Harmattan.

(C) L'Harmattan. Tous droits réservés pour tous pays.

La reproduction ou représentation de cet article, notamment par photocopie, n'est autorisée que dans les limites des conditions générales d'utilisation du site ou, le cas échéant, des conditions générales de la licence souscrite par votre établissement. Toute autre reproduction ou représentation, en tout ou partie, sous quelque forme et de quelque manière que ce soit, est interdite sauf accord préalable et écrit de l'éditeur, en dehors des cas prévus par la législation en vigueur en France. Il est précisé que son stockage dans une base de données est également interdit. 


\section{INTRODUCTION. LA SÉGRÉGATION MISE EN MOTS : QUELQUES RÉFLEXIONS SUR LA DIMENSION SOCIOLINGUISTIQUE DU PROCESSUS ${ }^{1}$}

\section{LES DIMENSIONS SOCIO-SPATIALES DU PROCESSUS SÉGRÉGATIF}

Le présent recueil centré sur la mise en mots de la ségrégation en situation urbanisée ${ }^{2}$ tente de rendre compte de la complexité des situations sociolinguistiques $^{3}$ que le chercheur est amené à rencontrer et à partir desquelles il souhaite mettre en place une intervention. Cette dernière dimension - voir Bulot (2011), Djerroud (2009) - est prégnante dans la mesure où elle constitue l'une des voies pour penser la durabilité urbaine et, en amont, l'action glottonomique nécessaire - et idéologiquement toujours ramenée à la part congrue - à toute action sur les habitats et, partant, sur les habitants. Ce qui caractérise de fait les espaces d'intervention de la sociolinguistique urbaine est qu'ils sont, certes, perçus/vécus comme plurilingues mais d'un plurilinguisme excluant, ostracisant voire discriminant et posant centralement les normes identitaires (dites urbanisées - voir sur ce concept Bulot et Ledegen, 2008) comme vecteurs/facteurs de positionnement énonciatif polarisant.

Rappelons brièvement (voir Bulot - 2001 et 2009 - pour plus de développement) comment la sociolinguistique urbaine pose la ségrégation. La sociolinguistique urbaine conçoit la ségrégation sous ses deux composantes polarisées : un état nécessaire des relations intra-urbaines et un processus permanent de construction des différents niveaux territoriaux de

${ }^{1}$ Gudrun Ledegen \& Thierry Bulot, PREFics EA 4246 / Université Européenne de Bretagne - Rennes 2 / GIS Pluralités Linguistiques et Culturelles.

${ }_{2}^{2}$ Il fait en quelque sorte écho non seulement à la dernière session des Journées Internationales de Sociolinguistique Urbaine, dont les débats sont parus dans Lebon-Eyquem, Bulot, Ledegen (2012) mais plus encore aux recherches présentées dans Bulot (2009 a et b), Lebon-Eyquem (2009), Laur (2009) où sont, entre autres, envisagés les liens dialectiques entre migrance et mobilité pour ce qu'ils permettent de distinguer les formes spatiogénétiques des processus ségrégatifs.

${ }^{3}$ Pour reprendre les propos de Michel Agier (1999: 15) que nous transposons à la sociolinguistique urbaine, nous dirons que ces situations sont définies par la combinaison des spatio-temporalités interactionnelles perçues et/ou vécues comme discursivement cohérentes, performatives, et contextualisées par leurs dimensions praxiques à d'autres espaces-temps d'interactions corrélés aux marquages spatio-langagiers de tous ordres. 
l'espace urbain. L'affrontement entre l'un et l'autre est en effet permanent dans la mesure où il s'agit à la fois - pour tous les types d'acteurs - de s'inscrire dans un système perçu comme donné, comme stable des relations entre les membres (groupes ou individus) de la communauté urbaine (l'espace citadin), en quelque sorte d'identifier à des lieux et des groupes, à des comportements socio-langagiers (l'espace urbain) et en même temps d'être l'instance de discours ${ }^{4}$ qui produit, modifie en interaction les représentations de l'espace, des rapports, aux lieux, aux langues (l'espace urbanisé), autrement dit, d'être dans une logique d'individuation sociolinguistique fondée et fondant la territorialisation, la mise à distance sociale dans la proximité métrique.

En tant qu'état et processus, la ségrégation donne lieu à des discours euxmêmes en relation dichotomique. En tant que concept, elle permet de comprendre la différenciation des espaces, la mise en place des frontières intra-urbaines en se fondant sur une dynamique de l'altérité, de mise à distance nécessaire. Elle est cependant insuffisante pour comprendre comment ces espaces, ces territoires, engagent une hiérarchisation des groupes et des personnes qui les occupent effectivement ou dont on dit qu'ils sont les seuls et remarquables et/ou légitimes occupants. Il convient de distinguer deux types de discours: ceux relevant spécifiquement de la ségrégation - parce qu'ils visent à mettre en place des frontières, des territoires, des sous-espaces distincts, à "mettre au dehors du groupe » initial (la communauté sociale urbanisée en l'occurrence) - et ceux relevant de la discrimination qui engagent - en appui sur l'espace perçu comme stable - la polarisation sociale des espaces, la spécialisation sélective des lieux qui reviennent aux groupes ségrégés, et enfin la polarisation négative de l'altérité. Ainsi, un discours ségrégatif pose de fait des lieux comme spécifiques de tel ou tel groupe social dans la ville, tandis qu'un discours discriminant, va poser - pour ces groupes - des attributs sociaux et langagiers justifiant $a$ posteriori la ségrégation.

\section{CORPUS ET/OU OBSERVABLES EN SOCIOLINGUISTIQUE URBAINE}

Cette livraison réunit ainsi diverses analyses de discours de ségrégation, prenant appui sur des corpus de presse, d'informateurs ou de documentaires ${ }^{5}$. Dans un premier temps, la dynamique ségrégative en cours dans la société mauricienne, qui voit arriver une nouvelle économie de « migration externe de luxe»(Yannick Bosquet-Ballah), ou dans la société allemande et

\footnotetext{
${ }^{4}$ Voir Bulot (2006) pour le rapport aux instances glottonomiques.

${ }^{5}$ Ces textes sont issus de présentations lors des JISU 2012 (La Réunion).
} 
La ségrégation mise en mots : quelques réflexions sur la dimension sociolinguistique... française, pour les jeunes qui vivent dans la marge (Sabine Bastian), est analysée dans les discours captés lors d'entretiens ou de documentaires menés dans les quartiers concernés. Dans un second temps, la nomination d'un groupe « autre », qu'il soit habitant de grands ensembles voués à la destruction (Frédéric Pugnière-Saavedra), mahorais ou comorien à La Réunion (Franck Marc), ou tsigane dans la presse française du début du siècle (Béatrice Turpin), prend implicitement appui sur un interdiscours ségrégant et normatif.

\section{ESPACE ET SÉGRÉGATION}

Yannick Bosquet-Ballah analyse les pratiques ségrégatives à l'œuvre dans la structuration de l'espace mauricien, en analysant par le biais d'une enquête de terrain, au sein des villages de Tamarin et Grande Rivière Noire, la mise en mots des espaces, des langues et des locuteurs et du marquage sociolinguistique. Historiquement, jusqu'à l'indépendance en 1968, la population mauricienne se caractérisait par une répartition dans l'espace principalement selon le critère ethnique, dont on peut encore voir des traces aujourd'hui. Un nouveau phénomène voit toutefois le jour, par l'augmentation de la «migration externe de luxe», créant de nouvelles organisations de l'espace, nettement ségrégatives : la population créole fait l'objet d'une mise à l'écart voire d'un confinement social et linguistique, alors que de nouveaux groupes venant d'Afrique du Sud s'installent de façon regroupée, en ségrégation tant sur le plan résidentiel, social, linguistique et économique : ils amènent certes le développement infrastructurel, ainsi que l'utilisation plus importante de l'anglais, mais déclenchent par ailleurs des tensions sur le plan linguistique comme économique. En complément à cette enquête, l'analyse des affichages urbains selon les quartiers de ces deux villages vient souligner cette ségrégation, confinant la pratique du créole exclusivement aux quartiers populaires et marginalisés, et instaurant l'anglais et le français en uniques pratiques en général, dans ces nouveaux quartiers ou lieux sur-modernes et prestigieux.

Dans son étude «Ces jeunes, qui vivent en marge : documentarisation et intervention sociolinguistique », Sabine Bastian analyse les raisons d'être de la situation précaire des jeunes d'un quartier en banlieue berlinoise, en passant en revue les différents facteurs de leur paupérisation et de leur (difficile) intégration : stigmatisation des habitants de ces quartiers; affaiblissement des réseaux d'information; réduction/ disparition de la mixité sociale; ségrégation scolaire... Prenant appui sur deux documentaires illustrant la précarité de la jeunesse allemande et française, Sabine Bastian étudie les dires des jeunes témoins qui parlent Kiezdeutsch ou « français contemporain des cités » (Goudaillier 2001), afin d'étudier les 

potentielles inter-traductions. Elle préconise l'étude approfondie de ce genre de corpus «pour éviter une banalisation voire une «surtraduction» des paroles des jeunes », dans ces documentarisations qui constituent un indispensable moyen de sensibiliser la société aux problèmes que rencontre la jeunesse marginalisée.

\section{NOMMER}

Frédéric Pugnière-Saavedra mène une approche par l'outil informatique, à l'aide de Prospéro, pour une étude socio-informatique et de Lexico 3 pour une analyse textométrique, sur des textes journalistiques (1995-2010), des avis de préfets et des discours de politiques. Il étudie ainsi la manière dont celui qui habite les grands ensembles émerge dans le discours sur la démolition et comment il prend la posture d'acteur agissant ou d'acteur subissant l'action de démolition. Ainsi, l'habitant se révèle peu mentionné dans les différents sous-corpus, mais tout de même majoritairement posé comme sujet agissant (1070 formes), plutôt qu'en tant qu'objet subissant (670 formes). Une axiologisation - souvent négative - se fait jour sur les lexèmes qui catégorisent l'habitant dans sa classe sociale et dans les conditions matérielles afférentes : classe populaire, conditions économiques faibles, constitution familiale, ethnicité, non insertion sociale. Ainsi, les différents corpus emploient des qualifications figées, préfabriquées, attendues $^{6}:$ le discours de la démolition est traversée par un "interdiscours, donnant la politique de rénovation urbaine comme la réhabilitation de l'espace laid, détérioré, insalubre pour permettre à l'habitant de vivre dans un espace qu'il espère plus harmonieux ».

Franck Marc examine la nomination du groupe des Mahorais et des Comoriens, "des identités autres », au sein de la société réunionnaise à travers la presse. Son corpus est constitué des trois principaux quotidiens de l'île de la Réunion: Le Journal de l'île, Le Quotidien, Témoignages sur l'année 2007. La nomination, désignation, qualification du groupe des Mahorais et des Comoriens dans ces discours sociaux les cantonne à la communauté migrante, allochtone, exclu de l'endogroupe réunionnais pourtant constitué dans un continuum culturel relativement stabilisé, et tacitement validé.

L'article «Inducteurs normatifs et discrimination. Un regard sur les Tsiganes dans les journaux du début du $\mathrm{XX}^{\mathrm{e}}$ siècle » est l'occasion pour

${ }^{6}$ Sollicitant fortement stéréotypes (Amossy \& Herschberg-Pierrot, 1997), prédiscours (Paveau, 2006), formules (Krieg-Planque, 2003), et routines (Wray, 2002). 
La ségrégation mise en mots : quelques réflexions sur la dimension sociolinguistique... Béatrice Turpin de procéder à l'étude d'un discours de presse du début du $\mathrm{XX}^{\mathrm{e}}$ siècle à travers les inducteurs normatifs (termes qui introduisent dans le discours un contenu normatif). Ces éléments de discours, et les thématiques qui y sont liées, révèlent les normes sociales, les représentations et les idéologies à cette époque déterminée : ainsi, la figure du «romanichel», instaurée comme «hors-la-loi », nomade, violent et volant (inducteurs juridiques) ; ou encore les inducteurs évaluatifs qui introduisent les normes morales, normes comportementales, et sont exposées de façon implicite par la mise en discours de leurs contraires... Béatrice Turpin met ainsi à jour les ingrédients de ce style normatif où les inducteurs ont une véritable fonction argumentative, «[posant] une inférence entre un discours donné et les discours circulants qui lui donnent sens ».

\section{BIBLIOGRAPHIE}

AGIER Michel, 1999, L'invention de la ville (Banlieues, townships, invasions et favelas), Editions des Archives Contemporaines, Paris, 176 pages.

AMOSSY Ruth \& HERSCHBERG-PIERROT Anne, 1997, Stéréotypes et clichés, Paris, Nathan université, Collection « $128 », 128$ pages.

BULOT Thierry \& LEDEGEN Gudrun, 2008, «Langues et espaces (Normes identitaires et urbanisation) », dans Cahiers de Sociolinguistique (Nouvelle Série) 13, Presses Universitaires de Rennes, Rennes, 5-14.

BULOT Thierry, 2001, «L'essence sociolinguistique des territoires urbains : un aménagement linguistique de la ville?», dans Sociolinguistique urbaine (Variations linguistiques : images urbaines et sociales), Cahiers de Sociolinguistique 6, Presses Universitaires de Rennes 2, Rennes, 5-11.

BULOT Thierry, 2006, La langue vivante (L'identité sociolinguistique des Cauchois), L'Harmattan, Paris, 223 pages.

BULOT Thierry, 2009, « La territorialisation sociolinguistique de la migrance (Propositions pour modéliser la discrimination des espaces en contexte plurilingue) », dans Formes \& normes sociolinguistiques (Ségrégations et discriminations urbaines), L'Harmattan (Collection Espaces Discursifs), Paris, 15-28. 
BULOT Thierry, 2009, « Pour une gestion durable des rapports entre le local et le global (intervention et sociolinguistique urbaine)», dans Sprache(n), Identität, Gesellschaft, Ibidem, Stuttgart, 63-72.

BULOT Thierry, 2011, «Espaces urbanisés durables et/ou espaces vulnérables en situations plurilingues. Mesures et questionnements sociolinguistiques ", dans Sociolinguistique urbaine - Identités et mise en mots, Martin Meidenbauer Verlag, München, 73-92.

DJERROUD Kahina, 2009, «Sociolinguistique prioritaire/de l'intervention : quelle(s) possibilité(s) d'action(s) à Alger», dans Sociolinguistique urbaine et développement durable urbain (Enjeux et pratiques dans les sociétés francophones et non francophones), Martin Meidenbauer Verlag, München, 59-72.

LAUR Elke, 2009, «La mise en mots d'une cité modèle située sur l'île de Montréal » ", dans Formes \& normes sociolinguistiques (Ségrégations et discriminations urbaines), L'Harmattan (Collection Espaces Discursifs), Paris, 181-208.

LEBON-EYQUEM Mylène, 2009, «Ségrégation spatio-linguistique et émergence des identités urbaines dans une ville de l'Est de La Réunion », dans Formes \& normes sociolinguistiques (Ségrégations et discriminations urbaines), L'Harmattan (Collection Espaces Discursifs), Paris, 159-180.

LEBON-EYQUEM Mylène, BULOT Thierry \& LEDEGEN Gudrun (Dirs.), 2012, Ségrégation, Normes et discrimination(s) (Sociolinguistique urbaine et migrance), Editions Modulaires Européennes \& Intercommunications (Collections Proximités), Fernelmont, 254 pages.

PAVEAU Marie-Annick, 2006, Les prédiscours. Sens, mémoire, cognition, Paris, Presses Sorbonne nouvelle, 252 pages.

WRAY Alison, 2002, Formulaic language and the lexicon, Cambridge, Cambridge University Press, 332 pages. 